\title{
El bienestar y sus aplicaciones en el campo de la Orientación
}

\author{
Irma Arguedas Negrini \\ Maestra en Orientación Escolar \\ Docente, Escuela de Orientación y Educación Especial \\ Facultad de Educación, Universidad de Costa Rica, Costa Rica \\ irma.arguedas@ucr.ac.cr \\ ORCID: https://orcid.org/0000-0002-9085-0185
}

Recepción: 01-10-2020 / Aceptación: 15-12-2020

\section{Resumen}

El trabajo ofrece fundamento teórico para la promoción del bienestar integral desde la Orientación y otras Ciencias de la Educación. Se describen su carácter multidimensional, diferentes modelos y las bases individuales y contextuales para su construcción.

El bienestar se define como el estado que resulta del equilibrio vital entre los desafíos y los recursos de las personas, lo que conduce al uso de las capacidades propias y la sensibilidad en el trato con los demás, desde los diferentes ámbitos de la integralidad humana. Se exponen similitudes y diferencias entre modelos de bienestar en relación con los ámbitos de los que se componen.

Asimismo, se discuten los elementos personales y contextuales que favorecen su construcción. En función del alcance del bienestar para todos, se resalta de relevancia de una sociedad en la que exista igualdad entre personas heterogéneas y diversas; reciprocidad y distribución equitativa de recursos y oportunidades.

Palabras clave: Bienestar, orientación, educación para el bienestar, sociedad equitativa, igualdad de oportunidades.

\section{Abstract}

The work offers a theoretical foundation for the promotion of comprehensive well-being from Guidance and other Education Sciences. Its multidimensional character, different models and the individual and contextual bases for its construction are described.

Well-being is defined as the state that results from the vital balance between people's challenges and resources, which leads to the use of their own capacities and sensitivity in dealing with others, from the different areas of human integrality. Similarities and differences between welfare models are exposed in relation to the areas of which they are composed.

Likewise, the personal and contextual elements that favor its construction are discussed. Depending on the scope of well-being for all, the relevance of a society in which there is equality between heterogeneous and diverse people stands out; reciprocity and equitable distribution of resources and opportunities.

Key words: Well-being, guidance, wellness education, equitable society. 


\section{Introducción}

El propósito de este trabajo es ofrecer fundamento teórico para las intervenciones de personas profesionales en Orientación y otras Ciencias de la Educación, en relación con la promoción del bienestar. Esto en respuesta a los propósitos de la disciplina de promover el desarrollo de las personas en todas sus áreas; prevenir problemáticas psicosociales e intervenir a nivel social en pro de las condiciones que favorecen la potenciación de todos.

En una reciente actualización al Plan de Estudios de la Carrera de Bachillerato y Licenciatura en Ciencias de la Educación con énfasis en Orientación que se imparte en la Universidad de Costa Rica, se establece como objeto de estudio "el desarrollo y el bienestar de las personas en las áreas personal, familiar, social, educativa, vocacional y comunal; mediante el empleo de los conocimientos teóricos que permiten facilitar procesos de Orientación que favorecen el aprendizaje para el crecimiento armónico de su ser y del entorno." (UCR/EOEE, 2019, p. 35). Esto representa un insumo que sustenta la llamada "Educación para el bienestar".

En el texto se hace referencia a lo que se entiende por bienestar, sus manifestaciones, diferentes modelos, así como las bases individuales y contextuales para su construcción.

\section{¿En qué consiste el bienestar?}

Bienestar es el estado que resulta del equilibrio vital entre los desafíos y los recursos de las personas, cuando estas cuentan con los recursos internos y externos para enfrentar sus desafíos; es cuando se puede decir que gozan de bienestar (Dodge, Daly, Huyton y Sanders, 2012). Tal equilibrio remite al funcionamiento en los diferentes ámbitos de la integralidad humana, que permite el uso de capacidades propias y la sensibilidad en el trato con las demás personas (Gleason y Narváez, 2019). De acuerdo con Mayordomo (2013).

El bienestar es un proceso multidimensional que se da en interacción entre las personas y el contexto socio-cultural e histórico; en él influyen tanto la variabilidad individual, como las constantes transformaciones en el entorno. Esta realidad conduce a la vitalidad y la autenticidad, aunque se pase por períodos de dificultad o malestar; implica encontrar significado en el desafío y que la persona dé lo mejor de sí, en función de metas y propósitos.

Diversas culturas hacen referencia al bienestar. A continuación, se presentan algunas palabras, consideradas intraducibles por Lomas (2016), pero todas referidas al bienestar, tal y como puede constatarse en el significado de cada una (ver figura 1).

Adicionalmente, es de relevancia mencionar el concepto de "buen vivir", el cual se sustenta en el paradigma de los pueblos indígenasoriginarios de América, y que puede considerarse sinónimo de los anteriores, aunque sí es traducible. Se refiere al sumak kawsay, que en quechua significa "vida en plenitud", y se refiere a la cultura y práctica cotidiana "de 


\begin{tabular}{|c|c|l|}
\hline Término & $\begin{array}{c}\text { Idioma al que } \\
\text { pertenece }\end{array}$ & \multicolumn{1}{|c|}{ Significado } \\
\hline Geborgenheit & Alemán & Sentir que se goza de protección y seguridad \\
\hline trygghet & Sueco & Sentido de satisfacción y de "estar en paz" \\
\hline sukha & Sánscrito & $\begin{array}{l}\text { Estado de felicidad duradero, que no depende } \\
\text { de situaciones específicas. }\end{array}$ \\
\hline ubuntu & Bantu & $\begin{array}{l}\text { Vivir con consideración y lealtad hacia las } \\
\text { demás personas }\end{array}$ \\
\hline ikigai & Japonés & La vida es buena y significativa \\
\hline
\end{tabular}

Figura 1. Cuadro resumen de términos utilizados en diferentes culturas para referirse al bienestar.

Nota. Adaptación propia con base en Lomas (2016).

respeto, armonía y equilibrio con todo lo que existe" (Huanacuni, 2010, p. 11).

En relación con este concepto, también es necesario indicar que un elemento central del "buen vivir" es el "buen convivir"; es decir, la armonía entre personas y con la Madre Tierra, el respeto a las visiones de mundo de cada cultura y la lucha por una sociedad en la que hay una justa repartición de los bienes (Tortosa, 2011).

Desde el punto de vista filosófico, el concepto de bienestar se ha abordado desde dos corrientes. Por un lado, la del hedonismo, también conocida como bienestar subjetivo, que plantea que la felicidad se alcanza mediante el placer. Por el otro, se encuentra la eudaimonía, que es el fundamento para el llamado bienestar psicológico, y visualiza la felicidad como la plenitud que se alcanza al poner en práctica las cualidades para alcanzar metas; vivir de acuerdo con el verdadero ser, tomar decisiones éticas ante situaciones difíciles y considerar los intereses y necesidades de las demás personas, en equilibrio con los propios. Esta última coincide con la visión humanista en que se sustenta el Plan de estudios de la carrera de Bachillerato y Licenciatura en Ciencias de la Educación con énfasis en Orientación, que se imparte en la Universidad de Costa Rica.

La perspectiva multidimensional del bienestar que fue presentada por Ryff (1989), a finales de la década de los 80 , enfatizó en la corriente eudaimónica al profundizar en una 
disposición vital y comportamientos motivados por el deseo de contribuir; y no solo por el propio placer (aunque no le quitó importancia al bienestar subjetivo). Esta autora distingue seis dimensiones: auto-aceptación, relaciones positivas con las demás personas, dominio de las variables del entorno, autonomía, crecimiento personal y propósito de vida.

Auto-aceptación significa conocer la multiplicidad de características, sentimientos y motivaciones, incluyendo limitaciones y aspectos no apreciados de la propia historia. Las relaciones positivas con las demás personas reflejan capacidad de amar, interacciones basadas en la confianza mutua y la empatía. El dominio de las variables del entorno es poder desenvolverse en diferentes contextos, de acuerdo con las propias necesidades y los propios valores. La autonomía es la regulación propia, sin presiones externas, para actuar o pensar de determinadas maneras. El crecimiento personal se refiere a la expresión de las potencialidades y a la introducción de cambios y mejoras. El propósito de vida es la dimensión relativa a la direccionalidad y el involucramiento en actividades que trascienden los intereses individuales.

Los estudios de Ryff guardan estrecha relación con la disciplina de Orientación, ya que como bien apuntan Machado y Bandeira (2012), están basados en postulados provenientes de enfoques humanístico-existenciales del campo de la salud mental y de la psicología del desarrollo, así como en la auto-realización de Maslow y la auto-actualización de Rogers.

\section{Desarrollo}

\section{Diferencias y semejanzas entre modelos de bienestar}

A lo largo de varias décadas ha habido propuestas de modelos de bienestar que incluyen variedad de ámbitos o tipos. Como se verá a continuación, se dan similitudes tanto como diferencias entre modelos, en relación con los ámbitos del bienestar de los que se componen.

Un modelo clásico en el campo de la Orientación -que se nutrió de otras disciplinas- es el denominado "Ser indivisible", propuesto por Myers y Sweeney (2004). Es un modelo holístico que se aboca a promover la calidad de vida y la longevidad, y se ha utilizado para fundamentar múltiples investigaciones e intervenciones. Mediante análisis factoriales se confirmó su validez y esa cualidad de indivisibilidad de las personas, en quienes interactúan aspectos que clasifican en esenciales, físicos, creativos, sociales y de adaptación.

La figura 2 es una representación de las fortalezas que integran dichos aspectos (ver figura 2).

Además del de Myers y Sweeney (2004), seguidamente se presentan otros cinco modelos. Uno de los más conocidos en Costa Rica es el llamado "Círculo del Bienestar" (Pereira, 1989), desarrollado por el Ministerio de Educación Pública con fines preventivos y de mejoramiento de la calidad de vida individual y social. Según la autora, este modelo se compone de los ámbitos: 


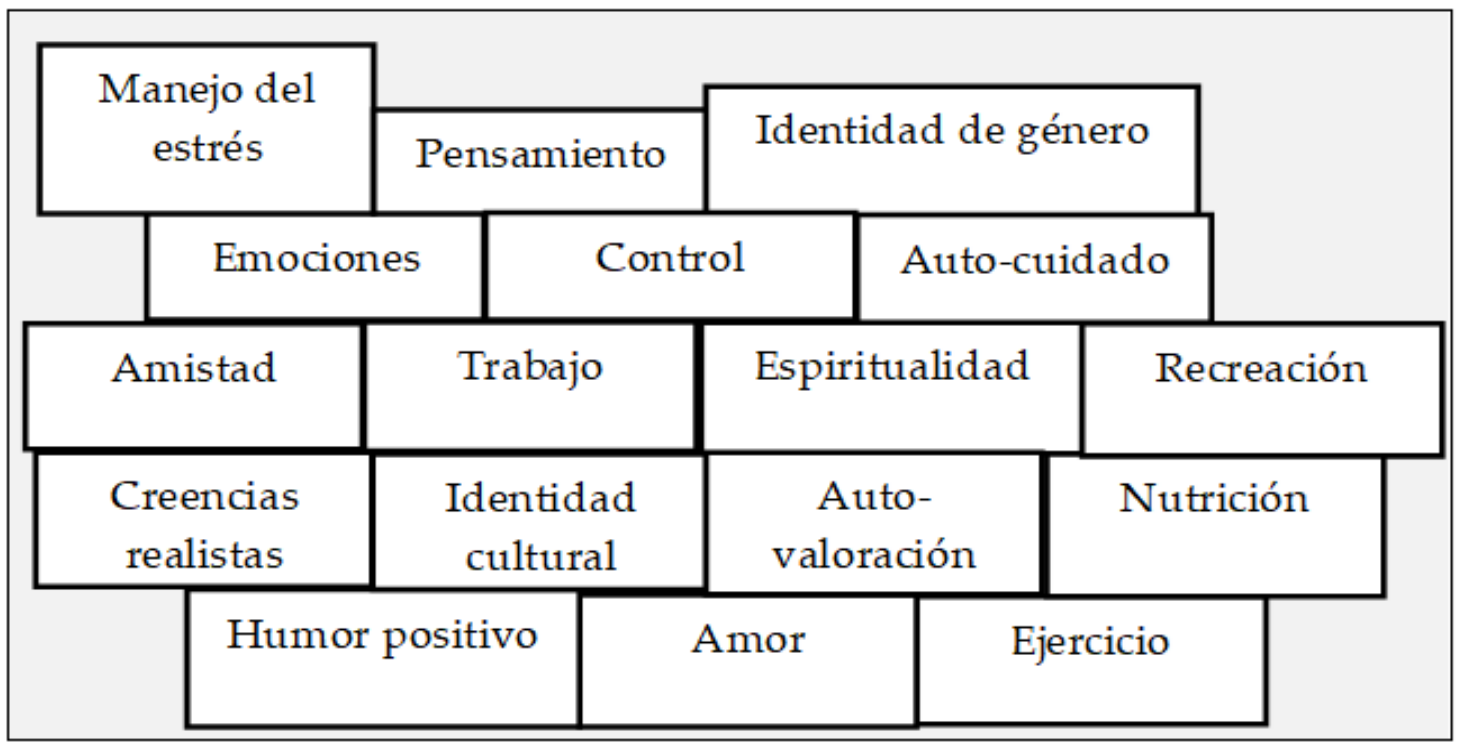

Figura 2. El ser indivisible

Nota. Adaptación propia con base en The Indivisible Self: An Evidence-Based Model of Wellness de Myers y Sweeney (2004).

1. Físico: Comprende factores que promueven la salud física y el compromiso con el medio ambiente.

2. Emocional: Incluye la creatividad, el reconocimiento de emociones, el disfrute de relaciones, de la naturaleza y del tiempo libre.

3. Social: Engloba la criticidad ante eventos nacionales e internacionales; los valores que facilitan relaciones solidarias, de respeto mutuo y de cooperación.

4. Ético: Integra valores personales, familiares y sociales con miras a una sociedad respetuosa, pacífica y armónica entre personas y con la naturaleza.

5. Vocacional: Visualiza el trabajo como derecho y fuente de satisfacción que debe realizarse de forma socialmente responsable.
Por su parte, Bisquerra (2013) plantea cinco tipos de bienestar:

1. Material: Se refiere al acceso a oportunidades y recursos básicos como la alimentación. Aquí opera la satisfacción por comparación con los demás, lo que tiene implicaciones negativas en sociedades consumistas y desiguales.

2. Físico: Incluye lo relativo al cuerpo y la salud como el ejercicio, el descanso y el evitar situaciones de riesgo con respecto a la alimentación; el uso de sustancias y la sexualidad. El bienestar físico también es influido por las condiciones ambientales y de la naturaleza.

3. Social: Refleja la calidad de las relaciones tanto a nivel interpersonal, como comunitario y político. Si las relaciones son 
favorables, hay más posibilidades para las personas de contribuir en cada uno de esos niveles, lo que favorece el bienestar general. A nivel político, la satisfacción y seguridad en el trabajo - sea este remunerado, doméstico o voluntario - es uno de los aportes más relevantes al bienestar. También es de gran importancia vivir en una comunidad hacia la que se siente orgullo y con los servicios básicos.

4. Emocional: Es un sentido de satisfacción con la propia vida, y predominio de cogniciones y reacciones emocionales positivas ante los acontecimientos. El predominio de emociones positivas es producto de lo que sucede en el contexto, y también de lo que la persona construye con su propio esfuerzo.

5. Profesional: Se relaciona directamente con el trabajo, por lo que su nivel depende en gran medida de si a la persona le agrada a lo que se dedica cada día; y tiene mucha influencia en los otros tipos de bienestar. En inglés, este tipo de bienestar se denomina career wellness.

Al igual que los otros modelos, las personas que proponen el modelo de bienestar derivado del Enfoque existencial de Orientación resaltan la naturaleza interdependiente de los ámbitos que lo componen. En el caso de este enfoque, los ámbitos son las cuatro dimensiones de la existencia humana (Leijssen, 2014):

1. Física o Umwelt, que significa medio ambiente. Se refiere a contar con seguridad, confort y salud; puede verse amenazada por la pobreza, la enfermedad y el deterioro físico.

2. Social o Mitwelt, que es estar en el mundo. Es el lugar que ocupa la persona en la sociedad y cómo se refleja en el reconocimiento, la pertenencia, la amistad y el cuido. Cuando hay un nivel bajo de bienestar social, la persona puede sentir rechazo, soledad y vergüenza.

3. Personal o Eigenwelt, que se traduce como mundo propio. Engloba características psicológicas y habilidades para enfrenar desafíos como la creatividad, el auto-conocimiento y el locus de control interno. Encierra los ideales de auto-trascendencia, el sentido de vida y la conciencia de la unidad con el todo.

4. Espiritual o Uberwelt, relativa al supramundo y a las conexiones con aquello que trasciende al ser. Es aplicable tanto a personas creyentes como ateas.

El enfoque existencial de Orientación plantea qué cambios en una dimensión generan modificaciones en otras; lo que ejemplifican indicando que, ante la pérdida de autonomía física, por enfermedad o declive en la vejez, es posible enriquecer la dimensión de espiritualidad. Asimismo, sostienen que, dependiendo de la cultura y de la persona, hay variaciones en el peso relativo de cada aspecto.

Es interesante que la perspectiva budista del bienestar (Sangasumana, 2019) es muy similar a la existencial. Desde dicha visión, el al- 
truismo y la mente compasiva son elementos importantes del bienestar, el cual integra los ámbitos material, social, mental y espiritual:

1. Material: Es la satisfacción con los recursos disponibles, incluyendo los que aportan seguridad y protección. La perspectiva budista incluye en este componente el disfrute de la naturaleza.

2. Social: Se refiere a la capacidad para establecer relaciones, las redes de apoyo y la equidad en las interacciones.

3. Mental: Se vincula con la libertad, el respetar y ser respetado; y la expresión de la creatividad.

4. Espiritual: Se refleja en la práctica de virtudes y creencias, la tolerancia y el altruismo. Su logro requiere el esfuerzo constante por transformar las creencias y acciones que alejan a la persona de la auto-trascendencia y la mente compasiva.

Específicamente, en relación con el bienestar durante el envejecimiento, Fullen (2019) propone un modelo de ocho componentes:

1. Contextual: Se refiere a la seguridad económica básica y al ambiente en el que se desenvuelve la persona que envejece. Si hay muchas privaciones a nivel individual y en la comunidad, se disminuyen las condiciones para un envejecimiento con bienestar.

2. Físico: Este componente es importante en todas las edades, pero en la adultez mayor cobra relevancia, debido a que es frecuente la presencia de enfermedad y dolor crónico; por lo que los apoyos para enfrentar esas condiciones son especialmente pertinentes.

3. Relacional: Engloba relaciones con amistades, parejas, familia y otros. El autor hace una diferencia entre socializar y desarrollar relaciones significativas al indicar que, aunque haya contacto con otras personas, puede experimentarse la soledad. Esto debido a que, la calidad de los recursos sociales es más importante que el número de personas que integran las redes de apoyo.

4. Emocional: Integra las percepciones y formas de interpretar que permiten conservar la esperanza y la estabilidad al enfrentar desafíos. Se manifiesta con el uso de estrategias actitudinales y sociales.

5. Cognitivo: Abarca la auto-eficacia y la implicación en actividades que promueven la estimulación cerebral y el aprendizaje. De acuerdo con el autor, la resiliencia se asocia con este tipo de bienestar.

6. Vocacional: En el marco de este modelo, se relaciona con hallar un propósito de vida en "cada capítulo de la adultez mayor" (p. 69), independientemente de si las actividades realizadas son remuneradas.

7. Espiritual: Permite encontrar sentido a las incertidumbres vitales e incorporar prácticas religiosas o no, que facilitan el 
afrontamiento. Es frecuente que las prácticas espirituales abran la oportunidad para participar en comunidades que representan un apoyo social.

8. Evolutivo: Es la construcción de una actitud saludable y realista acerca del proceso de envejecimiento, lo cual se asocia con longevidad. Refleja la creencia que, al en- vejecer, la vida sigue siendo valiosa y, por consiguiente, la persona se compromete con su propio cuidado.

En el siguiente cuadro se muestran semejanzas y diferencias entre los cinco modelos de bienestar, en relación con los ámbitos de los que se componen:

Tabla 1. Componentes del bienestar de acuerdo con cinco modelos

\begin{tabular}{|c|c|c|c|c|c|}
\hline & $\begin{array}{c}\text { Círculo } \\
\text { del } \\
\text { Bienestar }\end{array}$ & $\begin{array}{c}\text { Bisquerra } \\
\text { (2013) }\end{array}$ & $\begin{array}{c}\text { Enfoque } \\
\text { existencial de } \\
\text { Orientación }\end{array}$ & Budismo & $\begin{array}{c}\text { Durante en } \\
\text { envejecimiento } \\
\text { (Fullen, 2019) }\end{array}$ \\
\hline Material & & $\bullet$ & & $\bullet$ & \\
\hline Contextual & & & & & • \\
\hline Físico & $\bullet$ & $\bullet$ & $\bullet$ & & $\bullet$ \\
\hline Social & $\bullet$ & $\bullet$ & $\bullet$ & $\bullet$ & \\
\hline Relacional & & & & & $\bullet$ \\
\hline Emocional & $\bullet$ & $\bullet$ & & & $\bullet$ \\
\hline Personal & & & $\bullet$ & & \\
\hline Mental & & & & $\bullet$ & \\
\hline Cognitivo & & & & & $\bullet$ \\
\hline Vocacional & $\bullet$ & & & & $\bullet$ \\
\hline Profesional & & $\bullet$ & & & \\
\hline Ético & $\bullet$ & & & & \\
\hline Espiritual & & & $\bullet$ & $\bullet$ & $\bullet$ \\
\hline Evolutivo & & & & & - \\
\hline
\end{tabular}

Nota. Adaptación propia con base en Pereira (1989), Bisquerra (2013), Leijssen (2014), Sangasumana (2019) y Fullen (2019). 
Cabe agregar que entre los ámbitos de bienestar hay interacción, de forma que unos favorecen el enriquecimiento de los otros; y si se da un menoscabo en alguno, presenta afectación en los demás. Por ejemplo, Fullen (2019) reporta una relación entre el bienestar emocional y el relacional, así como entre el emocional y el cognitivo. Es por esto que, de acuerdo con el autor, las intervenciones profesionales para la promoción del bienestar deben reconocer la integralidad de la persona, y resaltar que la conexión entre ámbitos conduce a un fortalecimiento.

Asimismo, la interrelación y la importancia de cada componente pueden variar con la edad, la cultura y la clase social. El estudio de Blake, Nehrkorn y Patrick (2017) indica que las personas en condición de pobreza pueden tener mayores dificultades para atender su bienestar personal.

Si bien no existe una única trayectoria para el logro del bienestar, el marco conceptual acerca de los componentes y su interacción dinámica representa el fundamento para un abordaje, lo más integral e inclusivo posible, en la construcción en todos sus ámbitos. Se trata, según Fullen (2019), de una teoría multidimensional, holística, sinergística y enfocada en fortalezas.

\section{Elementos para la construcción del bienestar}

De acuerdo con Bisquerra (2013), el bienestar es un proceso que constantemente se está edificando para el beneficio individual y de la sociedad, a partir de elementos per- sonales y contextuales. Tanto a nivel personal como contextual, hay presencia de retos y de protecciones.

El proceso de construcción del bienestar es una interacción dinámica entre factores de riesgo, factores protectores y de justicia social, por lo que es esencial promover elementos personales y derribar las barreras que el medio presenta.

a. Elementos personales. Seligman (2016) aporta que, una de las características de los elementos personales que construyen bienestar es que las personas eligen desarrollarlos por sí mismos, y no en función de obtener los otros elementos. Así, por ejemplo, la persona con alto nivel de bienestar establece relaciones positivas porque está dentro de sus valores y no para obtener otras ganancias. No obstante, se cuenta con amplio sustento para afirmar que la calidad de las relaciones interpersonales tiene una profunda influencia en el bienestar.

Dentro de los elementos que el autor destaca se encuentran la implicación auténtica, la realización del potencial y encontrar sentido en la pertenencia y el servicio a algo que trasciende el yo. Agrega que ningún elemento por sí solo puede abarcar la integralidad del bienestar; asimismo, admite que es difícil lograr la presencia de todos los elementos, especialmente en un mismo momento. Cabe agregar que en lo relativo al bienestar, recibir apoyo es tan importante como ofrecerlo. 
Seligman (2016) destaca la importancia de hacer algo por otras personas, lo cual es una de las formas más certeras de enfrentar dificultades y construir una vida estimulante. Esto coincide con Mc Knight y Kashdan (2009), quienes exponen el propósito de brindar aspiraciones que organizan la experiencia vital. Los propósitos favorecen el establecimiento de metas y la identificación de recursos personales y externos; pueden compararse con una brújula que guía el accionar de las personas y está entretejida en su identidad. El propósito se asocia con la longevidad, con la salud y con el bienestar. Tener propósitos claros influye positivamente en la capacidad de las personas para enfrentar desafíos. La relación es bidireccional, ya que el propósito permite la edificación de fortalezas $y$, a su vez, las fortalezas o recursos personales favorecen el alcance de propósitos.

En la misma línea se ubican las premisas de la Logoterapia, uno de cuyos expositores indica que

Una vida plena es comprometerse con cada situación con todas las fuerzas. Lo decisivo es que no sea cualquier cosa de lo que nos ocupamos; sino que sea algo que percibimos como pleno de sentido. Cuanto mayor sea el número de situaciones vividas con sentido, tanto más plena llegará a ser la vida misma. Tanto más firme se siente el piso bajo los pies. (Längle, 2008, pp. 105-106)

Otros aportes relativos a los elementos individuales para la construcción del bienestar son los de Ciarrochi, Kashdan y Harris (2013). Sus estudios permitieron identificar siete pilares.

Para la definición de cada pilar se cumplió con dos criterios: que su existencia contara con fundamentación científica y que tuvieran uso práctico para facilitar cambios cognitivos y conductuales que conducen al bienestar (ver figura 3).

Es preciso señalar que, de acuerdo con Crespo (2016), en todas las edades se presenta el reto de la adaptación a cambios, tanto físicos como psicológicos y sociales; lo que demanda abordajes socioeducativos para el aprendizaje de estrategias, motivación y esfuerzo, que a su vez favorecen la resiliencia.

La autora añade que, para poder aplicar estrategias ante diferentes retos y metas, las personas tienen que creer en sus propias capacidades, así como tener percepción de control de algunos aspectos, como sus emociones, su disposición a esforzarse y su capacidad para enfrentar estresores.

b. Elementos contextuales. El bienestar se crea en el marco de la interacción entre ámbitos y sistemas en los que están inmersas las personas. Además de las fortalezas personales, se requiere del acceso a recursos y oportunidades en diferentes niveles, incluyendo los sociales, los organizativos y los del medio ambiente.

En referencia a las intervenciones orientadoras y socioeducativas, durante los primeros años de vida es necesario considerar que 


\begin{tabular}{|c|c|}
\hline Pilares & Beneficios \\
\hline $\begin{array}{l}\text { 1. Creencias funcionales acerca de sí } \\
\text { mismo, las otras personas y el mundo }\end{array}$ & $\begin{array}{l}\text { - Alcanzar logros } \\
\text { - Superar obstáculos } \\
\text { - Tener sentido de valía personal }\end{array}$ \\
\hline $\begin{array}{l}\text { 2. Atención plena (mindfulness) y toma } \\
\text { de conciencia }\end{array}$ & $\begin{array}{l}\text { - Conocer las propias emociones, procesos } \\
\text { mentales y estímulos externos } \\
\text { - Clarificar la mezcla de emociones que se } \\
\text { experimentan en un momento determinado }\end{array}$ \\
\hline 3. Toma de perspectiva & $\begin{array}{l}\text { - Empatía } \\
\text { - Tomar perspectiva con respecto a sí mismo }\end{array}$ \\
\hline 4. Valores & $\begin{array}{l}\text { - Reconocer los motivos de las propias } \\
\text { acciones } \\
\text { - Autonomía con respecto a las expectativas } \\
\text { de las otras personas }\end{array}$ \\
\hline 5. Aceptación de la experiencia & $\begin{array}{l}\text { - Coraje para enfrentar incertidumbres } \\
\text { - Valor para vivenciar estados de confusión }\end{array}$ \\
\hline 6. Control de la conducta & $\begin{array}{l}\text { - Logro de metas } \\
\text { - Implementación de la perseverancia } \\
\text { - Regulación de emociones }\end{array}$ \\
\hline 7. Desarrollo de habilidades cognitivas & $\begin{array}{l}\text { - Resolución de problemas } \\
\text { - Mejorar la concentración por medio de la } \\
\text { inhibición de estímulos irrelevantes }\end{array}$ \\
\hline
\end{tabular}

Figura 3. Cuadro resumen sobre los siete pilares del bienestar Nota. Adaptación propia con base en Ciarrichi, Kashdan y Harris (2013). 
las experiencias tempranas que promueven el bienestar son, sobre todo, los contextos y apoyos para el desarrollo integral. Uno de los ingredientes principales es la presencia de personas adultas que se comprometan con la formación de la niñez, y establezcan límites claros, amor incondicional, seguridad y oportunidades para la autorregulación; la realización de su potencial y el desarrollo de competencias emocionales y sociales (Ros, Filella, Ribes y Pérez, 2017; Gleason y Narváez, 2019).

Para la promoción del bienestar durante la adolescencia, Carpintero, López, Del Campo, Soriano y Lázaro (2007) recomiendan intervenciones educativas sistemáticas para edificar la auto-valoración y el auto-cuidado; así como la valoración y el cuido a las demás personas. Asimismo, dan importancia a la elección crítica de valores y la adopción de comportamientos coherentes con ellos.

Más adelante en la trayectoria vital, las personas adultas jóvenes se ven favorecidas - además de lo alcanzado en etapas anteriores - con oportunidades para desarrollar herramientas para el establecimiento de relaciones interpersonales significativas (no necesariamente de pareja). Para enfrentar las demandas a nivel laboral o de estudio; así como para introducir su propia estructura al dejar la estructura externa que dan el colegio o la familia (Stevanovic, Hoare, Mckenzie y O'Connor, 2017).

Para el bienestar durante la adultez y la adultez mayor, resaltan la relación entre la activi- dad constructiva y el bienestar, así como los efectos positivos de las oportunidades para una vejez generativa y con posibilidades de utilizar la creatividad. También es de destacar el vínculo estrecho entre temas de interés para la Gerontología, como lo son: el bienestar, la calidad de vida y el envejecimiento satisfactorio. La generatividad facilita la re significación de la propia muerte y la elaboración de pérdidas (Peixoto, 2017; Villareal, Hernándiz, Leal, Medina, y Vásquez, 2020).

Es indudable que, para el logro del bienestar son indispensables las condiciones que garanticen una vida democrática, seguridad ciudadana y respeto a los derechos humanos (Bisquerra, 2013). Esto lo reafirma Quijano (2013), al exponer que el buen vivir solo puede ser efectivo y tener sentido en una sociedad en la que existan igualdad entre personas heterogéneas y diversas; reciprocidad y distribución equitativa de recursos, y estén erradicadas la explotación y la violencia.

En este sentido, es importante conocer las necesidades de grupos particulares, especialmente de aquellos que enfrentan discriminación y navegan en un contexto con múltiples estresores, para así responder con oportunidades para la construcción del bienestar de todas las personas.

\section{Conclusiones}

La construcción del bienestar requiere de aprendizajes a lo largo de todo el ciclo vital, por lo que las personas requieren de las oportunidades en los diferentes ámbitos de bien- 
estar y desarrollo, para que puedan implicarse en la consecución de metas y puedan resistir imposiciones o riesgos que van en contra de su integridad.

Todos los tipos de bienestar pueden representar retos; y para todos es importante la construcción, identificación y uso de fortalezas. Aquí, la Educación y la Orientación cobran especial relevancia, al favorecer el desarrollo de habilidades de forma sistemática. También cobra relevancia la recomendación de Stevanovic, Hoare, Mckenzie y O'Connor (2017) de una "educación para el bienestar" y el desarrollo de las respectivas "habilidades para el bienestar"; las cuales concuerdan con las intervenciones orientadoras para la identificación y actualización de potencialidades; el conocimiento del medio natural y sociocultural, sus oportunidades y riesgos. Todo lo anterior es necesario para una acertada toma de decisiones y el establecimiento de metas que favorecen el bienestar personal y colectivo (UCR/EOEE, 2019).

Dada la multidimensionalidad del fenómeno del bienestar, el abordaje debe ser desde diferentes campos. En el caso de la Orientación, esta cuenta con herramientas específicas para hacer un aporte social, preventivo y en función del desarrollo integral de individuos, grupos, familias, comunidades e instituciones; por lo que, dentro de la identidad profesional debe ser central el quehacer relativo a la edificación de fortalezas y habilidades.

\section{Referencias}

Bisquerra, R. (2013). Cuestiones sobre bienestar. Cinco pilares para el desarrollo del bienestar personal, social y emocional. Madrid: Editorial Síntesis, S. A.

Blake, V. K.; Nehrkorn, A. M. y Patrick, J. H. (2017). Differential effects of health-promoting behaviors on wellbeing among adults. International Journal of Wellbeing, 7 (2), 28-42.

Carpintero, E.; López, F.; Del Campo, A.; Soriano, S. y Lázaro-Visa, S. (2007). El bienestar personal y social de los adolescentes y la prevención del malestar y la violencia: presentación y validación de un programa educativo. Revista de Investigación en Psicología, 10 (2), 29-41.

Ciarrochi, J.; Kashdan, T. B. y Harris, R. (2013). The Foundations of Flourishing. En: T. B. Kashdan y J. Ciarrochi (Eds.) Mindfulness, acceptance, and positive psychology: the seven foundations of well-being. pp. 1-29.

Crespo, M. T. (2016). Resiliencia, bienestar y aprendizaje a lo largo de la vida. International Journal of Development and Educational Psychology INFAD Revista de Psicología, 1 (2), pp. 161-170.

Dodge, R.; Daly, A.; Huyton, J. y Sanders, L. D. (2012). The challenge of defining wellbeing. International Journal of Wellbeing, 2 (3), 222-235. 
Fullen, M. (2019). Defining wellness in older adulthood: Toward a comprehensive framework. Journal of Counseling and Development, 97 (1), 62-74.

Gleason, T. R. y Narváez, D. (2019). Beyond resilience to thriving: Optimizing Child Wellbeing. International Journal of Wellbeing, 9 (4), 59-78.

Huanacuni, F. (2010). Buen Vivir / Vivir Bien. Filosofía, políticas, estrategias y experiencias regionales andinas. Lima, Perú: Coordinadora Andina de Organizaciones Indígenas - CAOI.

Längle, A. (2008). Vivir con sentido. Aplicación práctica de la logoterapia. Buenos Aires: Lumen.

Leijssen, M. (2014). Existential well being counseling. En G. Madison (Ed.) Emerging Practice in Focusing-Oriented Psychotherapy. Innovative Theory, Applications and Practice. Londres: Jessica Kinsley Publishers. pp. 142-157.

Lomas, T. (2016). Towards a positive cross-cultural lexicography: Enriching our emotional landscape through 216 'untranslatable' words pertaining to well-being. The Journal of Positive Psychology, 11 (5), pp. 546-558.

Machado, W. y Bandeira, D. R. (2012). Bem-estar psicológico: definição avaliação e principais correlatos. Estudos de Psicologia. Campinas, 29 (4), 587-595.

Mayordomo, T. (2013). Afrontamiento, resiliencia y bienestar a lo largo del ciclo vital. [Tesis doctoral]. Universitat de València.

Mc Knight, P. A. y Kashdan, T. B. (2009). Purpose in Life as a System that Creates and Sustains Health and Well-Being: An Integrative, Testable Theory. Review of General Psychology, 13 (3), 242-251.

Myers, J. y Sweeney, T. J. (2004). The Indivisible Self: An Evidence-Based Model of Wellness. Journal of Individual Psychology, 60 (3), 234-245.

Peixoto, C. (2017). Generatividad y bienestar psicológico en la vejez: un estudio con mayores voluntarios españoles y brasileños. [Tesis doctoral]. Universitat de València.

Pereira, M. T. (1989). El uso indebido de las drogas, su prevención: un reto para la educación. San José, Costa Rica: EUNED.

Quijano, A. (2013). ¿Bien vivir? Entre el “desarrollo” y la descolonialidad del poder. Contrapunto $(2), 83-93$.

Ros, A.; Filella, G.; Ribes, R. y Pérez, N. (2017). Análisis de la relación entre competencias emocionales, autoestima, clima de aula, rendimiento académico y nivel de bienestar en educación primaria. REOP, 28 (1), 8-18. 
Ryff, C. (1989). Happiness is everything, or is it? Explorations on the meaning of psychological wellbeing. Journal of Personality and Social Psychology, 57(6), 1069-1081.

Sangasumana, P. (2019). The Buddhist perspective on measuring wellbeing and happiness in sustainable development. International Journal of Multidisciplinary Development and Development, 6 (3), 243-248.

Seligman, M. E. P. (2016). Florecer. La nueva psicología positiva y la búsqueda del bienestar. México, D.F.: Océano Exprés.

Stevanovic, N.; Hoare, E.; Mckenzie, V. y O'Connor, M. (2017). Sustaining the use of Positive Education coping skills to meet the challenges of the emerging adulthood period. International Journal of Wellbeing, 7 (3), 39-55.

Tortosa, J. M. (2011). Vivir bien, Buen vivir: Caminar con los dos pies. Obets. Revista de Ciencias Sociales, 7 (1), 13-17.

Universidad de Costa Rica, Facultad de Educación, Escuela de Orientación y Educación Especial. Comisión de Plan de Estudios (2019). Plan de estudios de Bachillerato y Licenciatura en Ciencias de la Educación con énfasis en Orientación [folleto]. 